\title{
Naproxen affects osteogenesis of human mesenchymal stem cells via regulation of Indian hedgehog signaling molecules
}

Omar Salem ${ }^{1}$, Hong Tian Wang ${ }^{1}$, Abdulrahman M Alaseem ${ }^{1}$, Ovidiu Ciobanu¹, Insaf Hadjab ${ }^{1,3}$, Rahul Gawri ${ }^{1,4,5}$, John Antoniou ${ }^{1,2}$ and Fackson Mwale ${ }^{1,2^{*}}$

\begin{abstract}
Introduction: We previously showed that type X collagen, a marker of late stage chondrocyte hypertrophy (associated with endochondral ossification), is constitutively expressed by mesenchymal stem cells (MSCs) from osteoarthritis patients and this may be related to Naproxen (Npx), a nonsteroidal anti-inflammatory drug used for therapy. Hedgehog $(\mathrm{HH})$ signaling plays an important role during the development of bone. We tested the hypothesis that Npx affected osteogenic differentiation of human MSCs through the expression of Indian hedgehog (IHH), Patched-1 (PTC1) and GLI family members GLI1, GLI2, GLI3 in vitro.

Methods: MSCs were cultured in osteogenic differentiation medium without (control) or with $0.5 \mu \mathrm{M} \mathrm{Npx}$. The expression of collagen type $X$, alpha 1 (COL1OA1), alkaline phosphatase (ALP), osteopontin (OPN), osteocalcin (OC), collagen type I, alpha 1 (COL1A1) was analyzed with real-time reverse transcription (RT) PCR, and the ALP activity was measured. The osteogenesis of MSCs was monitored by mineral staining and quantification with alizarin red S. To examine whether Npx affects osteogenic differentiation through $\mathrm{HH}$ signaling, the effect of Npx on the expression of IHH, GLI1, GLI2, GLI3 and PTC1 was analyzed with real-time RT PCR. The effect of cyclopamine (Cpn), a HH signaling inhibitor, on the expression of COL1OA1, ALP, OC and COL1A1 was also determined.
\end{abstract}

Results: When MSCs were cultured in osteogenic differentiation medium, Npx supplementation led to a significant decrease in ALP gene expression as well as its activity, and had a tendency to decrease mineral deposition. It also decreased the expression of COLIA1 significantly. In contrast, the gene expression of COL IOA1 and OPN were upregulated significantly by Npx. No significant effect was found on OC expression. The expression of IHH, PTC1, GLII, and GLI2 was increased by Npx, while no significant difference was observed on GLI3 expression. Cpn reversed the effect of Npx on the expression of COL10A1, ALP, OPN and COL1A1.

Conclusions: These results indicate that Npx can affect gene expression during osteogenic differentiation of MSCS, and downregulate mineral deposition in the extracellular matrix through $\mathrm{HH}$ signaling. Therefore, Npx could affect MSC-mediated repair of subchondral bone in OA patients.

\section{Introduction}

Symptomatic osteoarthritis (OA), characterized by cartilage deterioration and osteophyte formation, is the most common joint disorder, affecting primarily the knees, hips, and hands, and the predominant symptom is pain [1]. It is the major reason for seeking medical care and

\footnotetext{
* Correspondence: fmwale@ldi.jgh.mcgill.ca

'Lady Davis Institute for Medical Research, Jewish General Hospital, McGill University, Montreal, Canada

2Division of Orthopaedic Surgery, McGill University, Montreal, Canada Full list of author information is available at the end of the article
}

accounts for most of the use of non-steroidal antiinflammatory drugs (NSAIDs) [2]. Clinical recommendations for the sympathetic treatment of OA include acetaminophen (Acet) and NSAIDs such as ibuprofen (Ibu), diclofenac (Dic), naproxen (Npx), and celecoxib (Cele) [3]. Unlike narcotics that target the central nervous system to alleviate pain, NSAIDs inhibit cyclooxygenase (COX) activity within the central nervous system and at the peripheral pain site to prevent the conversion of arachidonic acid into prostaglandins. Thus, NSAIDs can alter certain fundamental processes involved in the 
normal healing of injured tissues [4]. However, they are often inadequate and pain is the number one reason for undergoing joint replacement surgery [5].

Subchondral bone sclerosis and progressive cartilage degradation are widely considered as the hallmarks of OA. Despite the increase in bone volume fraction, subchondral bone is hypomineralized due to abnormal bone remodeling [6,7]. NSAIDs are used clinically to prevent ectopic bone formation that is also known as heterotopic ossification, and the efficacy of NSAIDs in the avoidance of heterotopic ossification has been documented in controlled clinical trials. Experimental studies also have documented the negative effects of NSAIDs on healing of skeletal tissues [8]. In earlier work, it was found that NSAIDs suppressed proliferation and induced cell death in cultured osteoblasts [9]. However, little is known about their effects on the subchondral bone repair in OA patients.

Mesenchymal stem cells (MSCs) are multipotent stromal cells capable of differentiating into multiple mesenchymal lineages, including osteogenic, chondrogenic, adipogenic, myogenic, and neurogenic lineages, under different conditions [10,11]. MSCs can differentiate to osteoblasts to form bone, while commitment of osteoprogenitor cells and differentiation into pre-osteoblasts, which eventually develop into mature osteoblasts, is a requisite [12]. To reverse or retard the degeneration of articular cartilage and repair the subchondral bone, MSCs can be employed in biological therapy for OA [13].

In the previous study, we found that NSAIDs can affect the expression of both hypertrophic and osteogenic genes in MSCs during expansion, and naproxen (Npx) showed a stronger effect on gene expression in MSCs than other drugs [14]. The effect of the drugs on gene expression in MSCs can influence the treatment of OA. Thus, it is important to understand whether $\mathrm{Npx}$ affects the osteogenesis of MSCs.

The Hedgehog $(\mathrm{HH})$ protein family has been found in all vertebrates, since their original discovery in Drosophila. Indian hedgehog $(\mathrm{IHH})$ regulates both chondrogenesis and endochondral bone formation $[15,16]$. It is known to stimulate bone formation via a positive feedback loop. Disruption of $\mathrm{HH}$ signaling results in severe skeletal abnormalities [17]. HH morphogens are secreted from the cells and bind to the receptor, Patched (PTC), to relinquish SMO from PTC suppression, thereby enabling activation of the GLI family of transcription factors, which are used as markers for $\mathrm{HH}$ signaling activity $[18,19]$. They are responsible for $\mathrm{HH}$-induced lineage commitment during MSC differentiation [20,21].

In this research, the effect of $\mathrm{Npx}$ on hypertrophy and osteogenesis of MSCs was studied. Because IHH signaling can affect the osteogenesis of MSCs and other osteogenesis-related signaling pathways [21-23], the effect of Npx on IHH signaling was also studied to elucidate the mechanisms involved.

\section{Materials and methods}

\section{Source and expansion of stem cells}

Normal human MSCs were obtained from Lonza Walkersville Inc (Walkersville, MD, USA). According to the supplier, these cells were harvested from healthy human bone marrow and were positive for CD105, CD166, CD29 and CD44, but negative for CD14, CD34 and CD45. All cells were expanded in DMEM supplemented with $10 \% \mathrm{FBS}, 100 \mathrm{U} / \mathrm{mL}$ penicillin, and $100 \mu \mathrm{g} / \mathrm{mL}$ streptomycin and were used within four passages. The culture medium and the supplement were from Wisent Inc (St-Bruno, QC, Canada).

\section{Cell culture}

In every well of a six-well plate (Sarstedt, QC, Canada), $5 \times 10^{5}$ MSCs were plated and cultured in expansion medium overnight. The floating cells were removed and the attached cells were cultured until the confluence was more than $90 \%$. Then the cells were cultured in osteogenic differentiation medium for 3 days to allow the cells to adapt to the new environment. Afterwards, the cells were cultured in $3 \mathrm{~mL} /$ well of osteogenic differentiation medium with $0.5 \mu \mathrm{M}$ Npx (Sigma-Aldrich, Oakville, ON, Canada). The cells cultured without Npx were used as control cells. To test the effect of cyclopamine (Cpn) on gene expression in MSCs, $0.5 \mu \mathrm{M}$ cyclopamine (Sigma-Aldrich) was dissolved in the culture medium. The osteogenic differentiation medium was prepared with high-glucose DMEM containing 10\% FBS, $0.1 \mu \mathrm{M}$ dexamethasone, $10 \mathrm{mM} \beta$-glycerophosphate, $50 \mu \mathrm{M}$ L-ascorbic acid, 100 units $/ \mathrm{mL}$ penicillin, and $100 \mu \mathrm{g} / \mathrm{mL}$ streptomycin.

\section{Total RNA isolation}

After MSCs were cultured for 3, 6 and 12 days, they were washed with PBS and total RNA was extracted using Trizol reagent (Invitrogen, Burlington, ON, Canada) according to the protocol from the supplier $[11,14]$.

\section{Reverse transcription and real-time PCR}

First, $1 \mu \mathrm{g}$ total RNA isolated from the cells was digested with DNase I (Invitrogen) according to the protocol of the supporter. Then, the purified RNA was reversetranscribed as described previously [11,14]. Briefly, $1 \mu \mathrm{g}$ RNA was mixed with random primers (final concentration $0.15 \mu \mathrm{g} / \mu \mathrm{L}$ ), dNTP mixture (final concentration $0.5 \mathrm{mM}$ ), and DEPC-treated distilled water with a total volume of $12 \mu \mathrm{L}$. After the solution was incubated at $65^{\circ} \mathrm{C}$ for 5 minutes, it was mixed with a first-strand 
buffer, Dithiothreitol, RNaseOUT, and Superscript II reverse transcriptase with a final volume of $20 \mu \mathrm{L}$. Then, the solution was incubated at $45^{\circ} \mathrm{C}$ for $50 \mathrm{mi}$ nutes to perform the reverse transcription and then at $70^{\circ} \mathrm{C}$ for 15 minutes to inactivate the reverse transcriptase. For LightCycler real-time PCR, a master mix of the following reaction components was prepared with the final concentrations at $10 \mu \mathrm{L}$ SYBER PCR master mix (Qiagen, Mississauga, ON, Canada), $8 \mu \mathrm{L}$ distilled water, $0.5 \mu \mathrm{L}$ forward primer (final concentration $0.25 \mu \mathrm{M}$ ), and $0.5 \mu \mathrm{L}$ reverse primer (final concentration $0.25 \mu \mathrm{M}$ ). To each $19 \mu \mathrm{L}$ master mix, $1 \mu \mathrm{L}$ of cDNA was mixed as a PCR template. The sequences of primers are in Table 1 . The reaction conditions included one cycle of PCR initial activation step $\left(95^{\circ} \mathrm{C}\right.$ for 15 minutes, $20^{\circ} \mathrm{C} / \mathrm{s}$ ramp rate), 45 cycles of amplification and quantification $\left(94^{\circ} \mathrm{C}\right.$ for $15 \mathrm{~s}, 57^{\circ} \mathrm{C}$ for $30 \mathrm{~s}, 72^{\circ} \mathrm{C}$ for $30 \mathrm{~s}$ ), one cycle of melting curve $\left(65^{\circ} \mathrm{C}\right.$ to $95^{\circ} \mathrm{C}$ with heating rate of $0.1^{\circ} \mathrm{C} / \mathrm{s}$ with a continuous fluorescence measurement), and a final cooling step to $4^{\circ} \mathrm{C}$.

The crossing points (CPs) were determined by the Light-Cycler software 3.3 (Roche Diagnostics, Indianapolis, IN, USA) and were measured at constant fluorescence level. The ratio of gene expression relative to GAPDH

Table 1 Primer sequences

\begin{tabular}{|c|c|c|}
\hline Gene & Sequence & Size (bp) \\
\hline \multirow[t]{2}{*}{$A L P$} & Forward (1397-1416): CCACGTCTTCACATTTGGTG & 196 \\
\hline & Reverse (1573-1592): AGACTGCGCCTGGTAGTTGT & \\
\hline \multirow[t]{2}{*}{ COLIA1 } & Forward (3982-4001): GAGAGCATGACCGATGGATT & 178 \\
\hline & Reverse (4140-4159): CCTTCTTGAGGTTGCCAGTC & \\
\hline \multirow[t]{2}{*}{ COLIOA1 } & Forward (1670-1690): AATGCCTGTGTCTGCTTTTAC & 130 \\
\hline & Reverse (1779-1799): ACAAGTAAAGATTCCAGTCCT & \\
\hline \multirow[t]{2}{*}{ GAPDH } & Forward (113-133): TGAAGGTCGGAGTCAACGGAT & 181 \\
\hline & Reverse (273-293): TTCTCAGCCTTGACGGTGCCA & \\
\hline \multirow[t]{2}{*}{ GL/1 } & Forward (676-695): AAGCGTGAGCCTGAATCTGT & 189 \\
\hline & Reverse (845-864): CAGCATGTACTGGGCTTTGA & \\
\hline \multirow[t]{2}{*}{ GLI2 } & Forward (199-218): CGACACCAGGAAGGAAGGTA & 203 \\
\hline & Reverse (382-401): TGCACAGAACGGAGGTAGTG & \\
\hline \multirow[t]{2}{*}{ GLI3 } & Forward (2285-2304): CTTTGCAAGCCAGGAGAAAC & 163 \\
\hline & Reverse (2428-2447): TTGTTGGACTGTGTGCCATT & \\
\hline \multirow[t]{2}{*}{$\mathrm{IHH}$} & Forward (519-538): CGGCTITGACTGGGTGTATT & 219 \\
\hline & Reverse (718-737): AAAATGAGCACATCGCTGAA & \\
\hline \multirow[t]{2}{*}{ OC } & Forward (20-39): TGAGAGCCCTCACACTCCTC & 151 \\
\hline & Reverse (170-151): CGCCTGGGTCTCTTCACTAC & \\
\hline \multirow[t]{2}{*}{ OPN } & Forward (759-778): TGAAACGAGTCAGCTGGATG & 162 \\
\hline & Reverse (920-901): TGAAATTCATGGCTGTGGAA & \\
\hline \multirow[t]{2}{*}{ PTC1 } & Forward (1460-1479): TCAGCAATGTCACAGCCTTC & 248 \\
\hline & Reverse (1688-1707): GTCGTGTGTGTCGGTGTAGG & \\
\hline
\end{tabular}

as the reference gene was determined by the following equation $[11,14]$ :

Relative ratio $=\frac{2^{\Delta C \mathrm{P}_{\text {target }}(\text { control-sample })}}{2^{\Delta C \mathrm{P}_{\text {reference }}(\text { control-sample })}}$

\section{Alkaline phosphatase activity}

After MSCs were cultured in osteogenic differentiation medium with or without $\mathrm{Npx}$ for 3,6 , and 12 days, the cells were lysed and ALP activity was assayed with StemTAG ${ }^{\mathrm{m}}$ Alkaline Phosphatase Activity Assay Kit (Colorimetric) (Cell Biolabs Inc, San Diego, CA, USA) according to the protocol from the supplier.

\section{Mineralization analysis with alizarin red S}

Osteogenic differentiation was monitored by mineral deposition with alizarin red S staining as previously described [11]. To quantify the matrix mineralization, alizarin red $\mathrm{S}$ was extracted with $1 \mathrm{~mL} /$ well $100 \mathrm{mM}$ cetylpyridinium chloride (Sigma-Aldrich) and measured at $570 \mathrm{~nm}$.

\section{Statistical analysis}

Statistical analysis was performed using one-way analysis of variance (ANOVA), followed by Fisher's protected least significant difference post hoc test, using Statview (SAS Institute, Inc). The results of three experiments with MSCs from three different donors were assessed, and the values were reported as mean $\pm \mathrm{SD}$. The significance was defined as a P-value $<0.05$.

\section{Results}

Message expression for various matrix protein genes was analyzed after culturing MSCs in osteogenic conditions supplemented with or without Npx for 3, 6 and 12 days. COL10A1 is a marker gene for hypertrophic chondrocyte differentiation $[24,25]$. At day 6 , the COL10A1 message was increased significantly with Npx supplementation in the culture medium $(P=0.007)$, while no significant difference was found at days 3 and 12 (Figure 1A). Alkaline phosphatase $(A L P)$, osteopontin $(O P N)$, osteocalcin $(O C)$ and COL1A1 are important genes that define the osteogenesis of MSCs [24-26]. To test the effect of Npx on osteogenesis of MSCs, the expression of these genes was examined after MSCs were cultured in osteogenic differentiation medium with or without $\mathrm{Npx}$ for 3, 6 and 12 days. When MSCs were cultured with Npx for 6 and 12 days, message levels of $A L P$ were decreased significantly compared with control $(P=0.007$ on day 6 ; $P=0.001$ on day 12 ), while no significant difference was observed on day 3 (Figure 1B). Npx increased OPN expression significantly on day $6(P=0.006)$ and day 12 


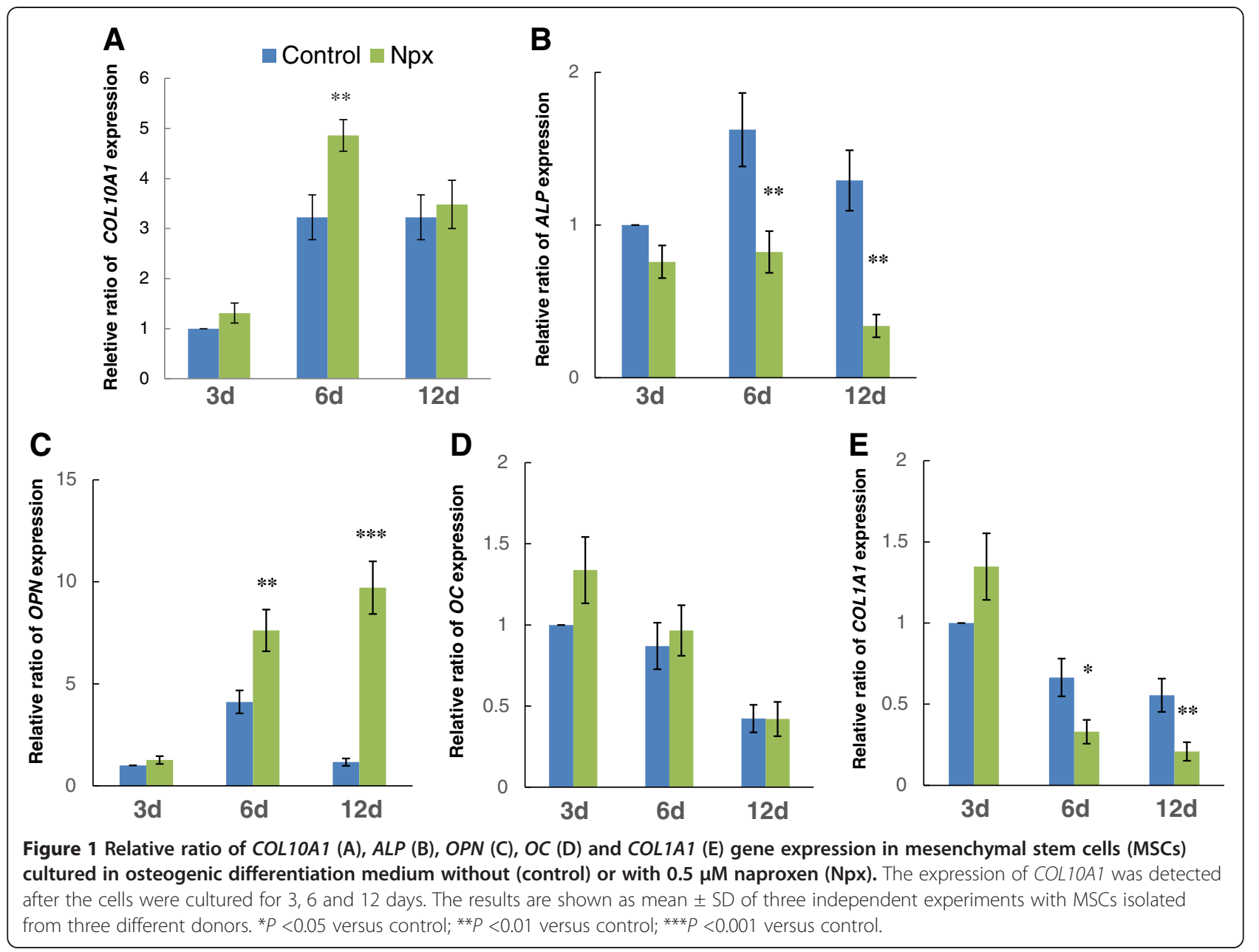

$(P<0.001)$ respectively, while no significant difference was observed on day 3 (Figure 1C). Treatment with Npx did not result in a significant change in $O C$ expression (Figure 1D). Npx decreased the expression of COL1A1 significantly on day $6(P=0.013)$ and day 12 $(P=0.007)$, while no significant effect was observed on day 3 (Figure 1E).

As Npx suppressed $A L P$ gene expression (Figure 1B), we next tested the effect of Npx on ALP activity (Figure 2). Npx significantly decreased ALP activity when MSCs were cultured with Npx for 6 days $(P=0.03)$ and 12 days $(P=0.004)$, but did not significantly decrease ALP activity on day 3 (Figure 2).

In order to verify that MSC cultured in osteogenic differentiation medium with $\mathrm{Npx}$ for 21 days results in the deposition of mineral, they were visualized by staining the cells with alizarin red $\mathrm{S}$, which detects calcium deposition. Red staining was present throughout the cultures (Figures 3A,B). With Npx, there appeared to be a decrease in the extent of matrix mineralization. When alizarin red $\mathrm{S}$ was extracted from the matrix, mineral deposition in the presence of Npx decreased although not significantly compared with control cells (Figure 3C).

IHH signaling is an important pathway in osteogenic differentiation, and is defined by IHH, GLI1, GLI2, GLI3 and PTC1 genes $[18,19]$. The effect of Npx on the expression of these genes was therefore assessed. When the cells were cultured in osteogenic differentiation medium with Npx, the expression of $I H H$ increased significantly on day $6(P<0.001)$ and day $12(P=0.006)$ compared with that in control cells (Figure 4A). This difference was not observed on day 3 . The expression of PTC1 $(P=0.006)$, GLI2 $(P=0.005)$ increased significantly on day $12 \mathrm{com}-$ pared with that in control cells (Figure 4B,D), while the expression of GLI1 increased significantly on both day 3 $(P=0.001)$ and day $12(P=0.003)$ (Figure $4 C)$. No significant effect of Npx on the expression of GLI3 was observed (Figure 4D).

Cpn can downregulate $\mathrm{HH}$ signaling pathway activity by binding directly to SMO, a key component of the $\mathrm{HH}$ signaling pathway $[27,28]$. To determine whether the 


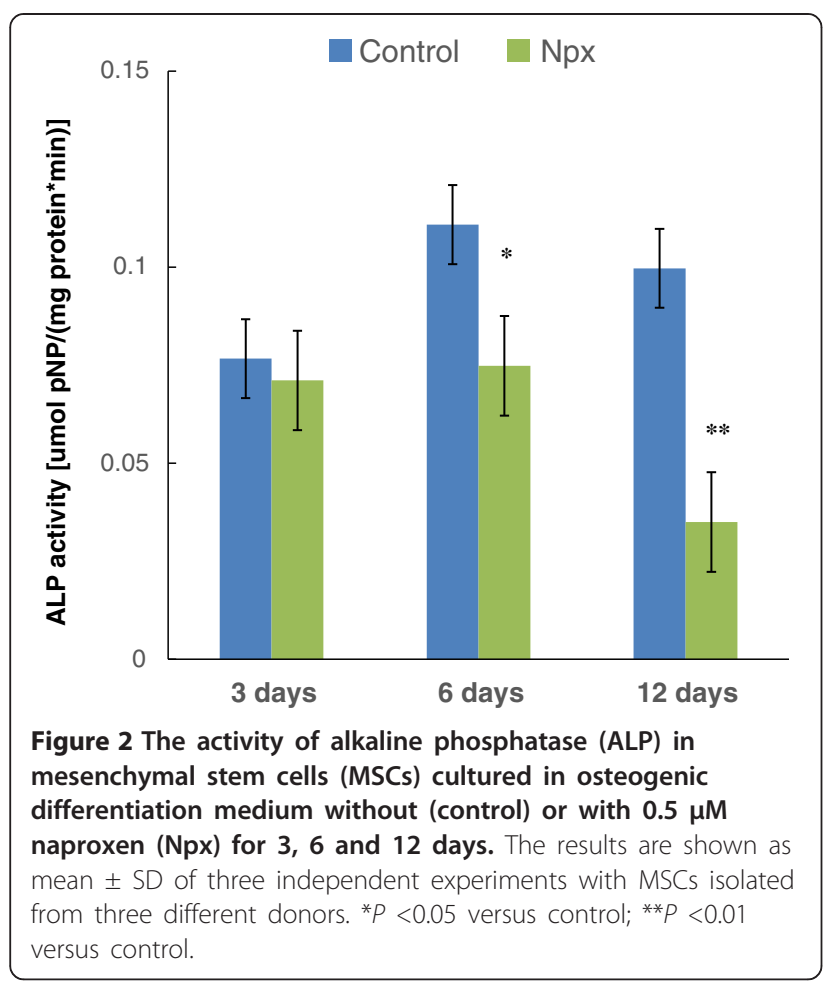

effect of Npx on the expression of COL10A1, ALP, OPN and COL1A1 was directly through the $\mathrm{HH}$ signaling pathway, MSCs were cultured in osteogenic differentiation medium with $\mathrm{Npx}$ alone or both $\mathrm{Npx}$ and $\mathrm{Cpn}$. On day 6 , the expression of COL10A1 with cyplocamine supplementation decreased significantly compared with Npx alone $(P=0.04)$ (Figure 5A). ALP expression was increased significantly with $C p n$ supplementation compared with Npx alone on day $6(P=0.003)$ and day 12 $(P=0.002)$ (Figure $5 \mathrm{~B})$. Compared with Npx alone, the expression of $O P N$ was decreased significantly by $\mathrm{Cpn}$ on both day $6(P<0.001)$ and day $12(P=0.007)$ (Figure $5 C)$. The expression of COL1A1 was upregulated by $\mathrm{Cpn}$ on day $6(P=0.002)$ and day $12(P=0.04)$. The significant difference was not observed for either COL10A1 (Figure 5A) or $A L P$ (Figure $5 \mathrm{~B}$ ) expression with $\mathrm{Cpn}$ supplementation on day 12. Thus Npx appeared to affect the expression of COL1OA1, ALP, OPN and COL1A1 through $\mathrm{HH}$ signaling in MSCs. Preliminary studies showed that Cpn alone had no effect on COL10A1, ALP, OPN and COL1A1 expression (data not shown), and because the results of culturing MSCs in osteogenic differentiation medium alone were conducted in Figure 1, these were not included in order to simplify the figures.

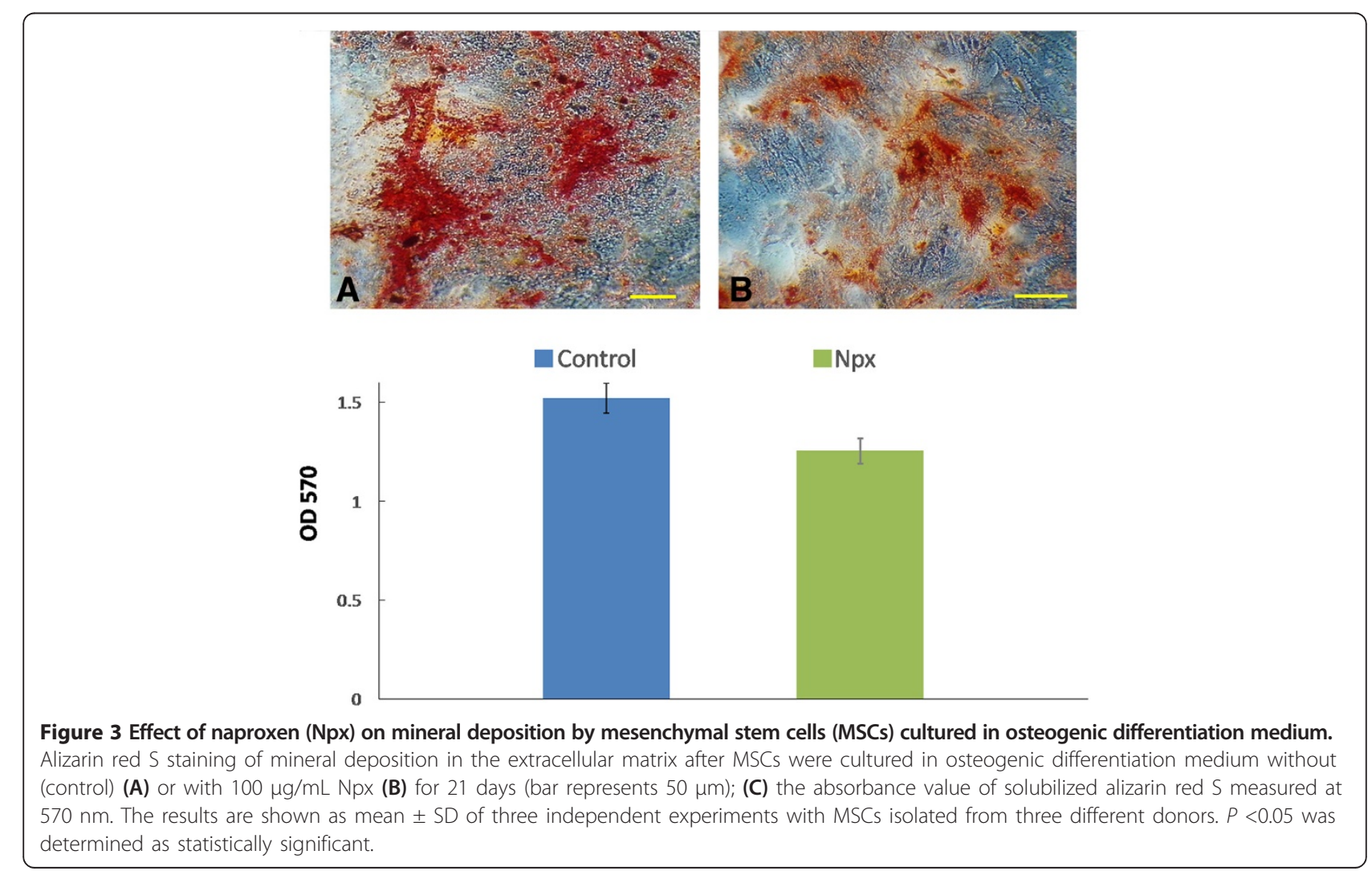




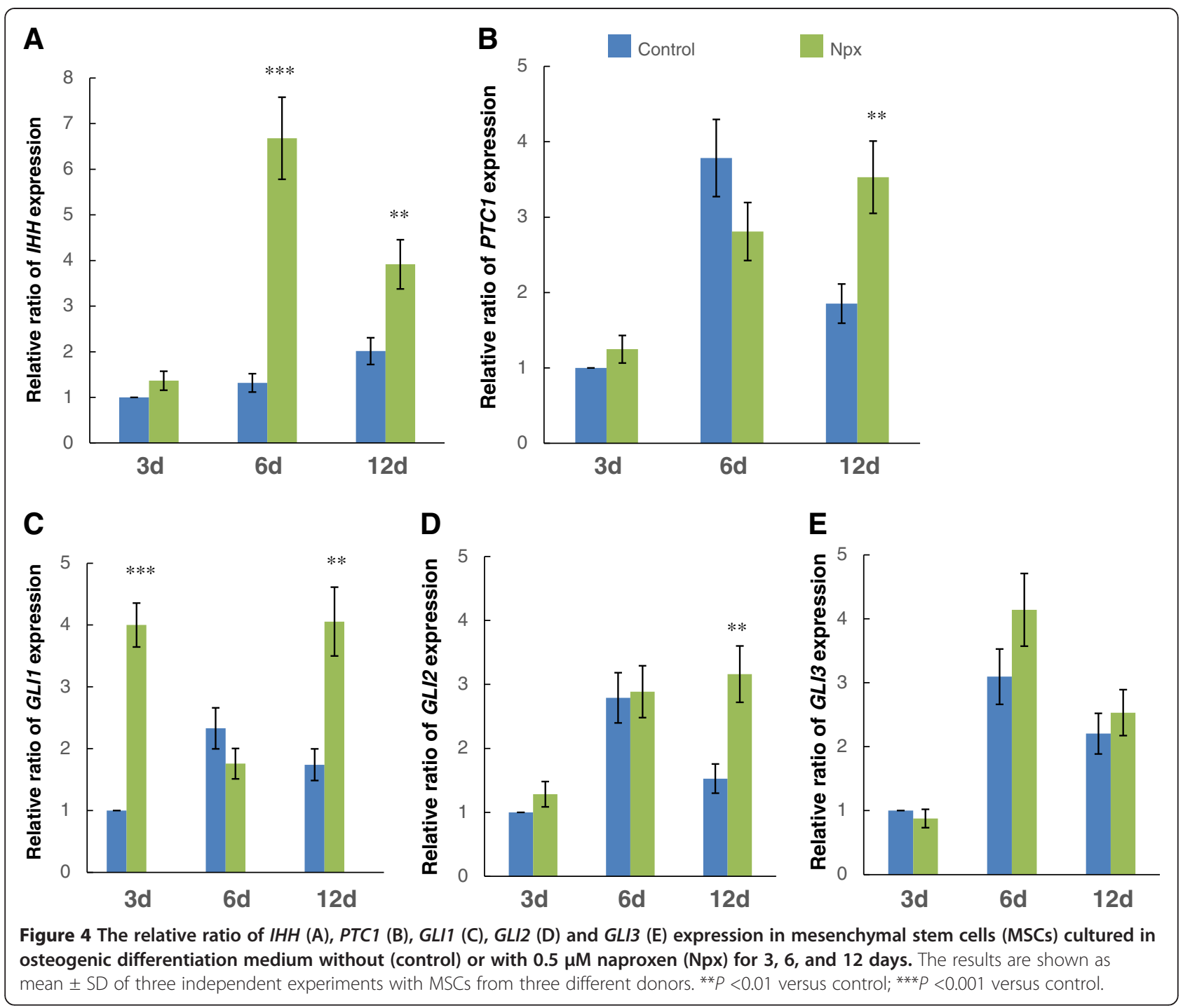

\section{Discussion}

If MSCs are to be used to stimulate bone repair in the presence of NSAIDs in the synovial fluid, it is essential that NSAIDs do not interfere with osteogenic differentiation. The results of the present work indicate that Npx can induce the expression of COL10A1 and OPN, and downregulate the expression of $A L P$ and COL1A1 through activation of $\mathrm{HH}$ signaling. The downregulation of $A L P$ gene expression led to the suppression of its activity and decreased mineral deposition. Osteogenesis occurs through an endochondral process involving cellular hypertrophy and mineralization in a manner analogous to the growth plate. Type $\mathrm{X}$ collagen is a marker of hypertrophy, whereas ALP, OC, OPN and COL1A1 are bone matrix proteins synthesized by osteoblasts [24-26]. Mineralization is a functional endpoint reflecting advanced osteogenesis. Achieving increased expression of OC, OPN, COL1A1, as well as ALP activity and mineralization is therefore an essential requisite for bone repair. Thus Npx has the potential to interfere with MSCs in repairing bone.

The effect of anti-inflammatory drugs on the osteogenesis of MSCs has been reported previously in other studies. It was reported that the osteogenic potential of MSC is inhibited/delayed by treatment with highdose NSAIDs under inflammatory conditions, while no significant effects were observed in non-inflammatoryconditioned MSCs [29]. In another experiment, it was reported that NSAIDs can inhibit bone formation via blockage of MSC chondrogenic differentiation - an important intermediate phase in normal endochondral bone formation - but not the osteogenesis of MSC [30]. In our study, Npx had a tendency to downregulate matrix mineralization. Furthermore, its effect on the expression of OPN and COL1A1 could affect the function of bone. In addition to their effects on osteogenesis, high-dose and 

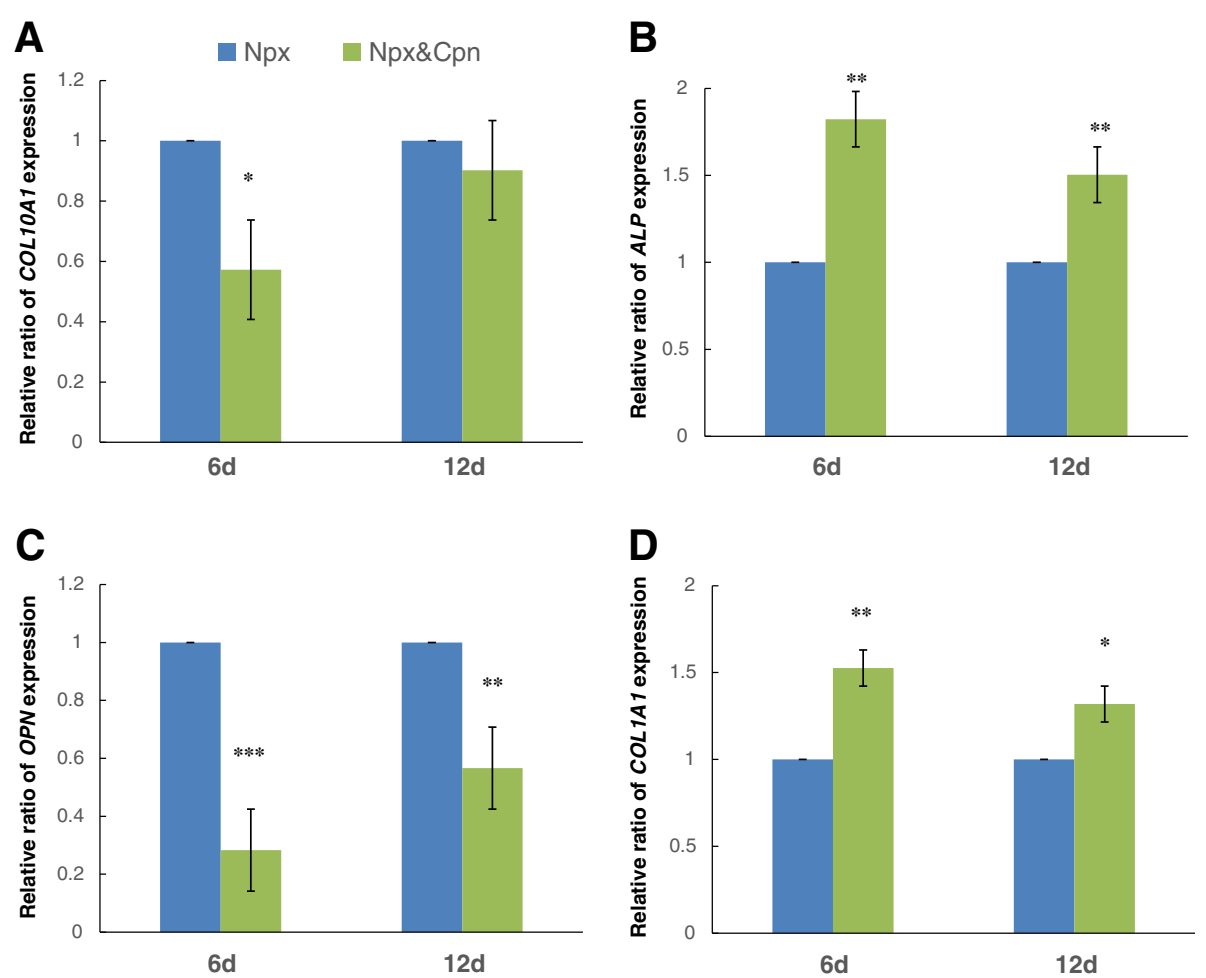

Figure 5 The effect of the Hedgehog $(\mathrm{HH})$ signaling pathway inhibitor cyclopamine (Cpn) on the expression of COL10A1 (A), ALP (B), OPN (C) and COL1A1 (D) in mesenchymal stem cells (MSCs) cultured in osteogenic differentiation medium with $0.5 \mu \mathrm{M}$ naproxen (Npx) for 6 and 12 days. The results are shown as mean \pm SD of three independent experiments with MSCs from three different donors. ${ }^{*} P<0.05$; **P $<0.01$ cells cultured with both cyclopamine (Cpn) and naproxen (Npx) versus cells cultured with Npx only.

long-term administration of NSAIDs has been shown to induce adipogenesis in stem cells. For instance, Indomethacin can stimulate adipogenesis of MSCs [31]. However, the effect of Npx on adipogenesis is not known. Interestingly, Npx was shown to have anti-obesity effects in animals when injected at a high dose [32].

A decrease in the expression of osteogenic markers and functional mineralization, and an increase in the expression of hypertrophic chondrocyte markers was observed after prolonged culture with Npx. During hypertrophy, cells enlarge in many tissues such as the growth plate, muscle and cartilage, which also occurs in conditions of degeneration, such as OA. However, we did not observe any appreciable increase in cell volume. It has been reported that calcium/calmodulin-dependent kinase II (CamkII) is an essential component of intracellular signaling pathways regulating chondrocyte maturation, and it can induce cell hypertrophy through a branched set of effector pathways including the transcriptional regulators Runx2 and Mef2c [33,34]. Activation of CamkII activity can result in premature hypertrophic gene expression, with no cell swelling. Furthermore, chondrocytes can display morphological changes consistent with hypertrophy, without upregulating the expression of hypertrophic genes
[33,34]. Thus, the hypertrophic program may be more complex than previously thought.

Npx affected the expression of IHH, PTC1, GLI1 and GLI2 genes belonging to the $\mathrm{HH}$ signaling pathway, and affected the expression of osteogenic genes in MSCs at different time points. This might be the result of the interaction of different proteins involved in $\mathrm{HH}$ signaling [22]. The upregulation of IHH, GLI1 and GLI2 can increase the $\mathrm{HH}$ signaling pathway positively, while the upregulation of PTC1 has a negative effect on the $\mathrm{HH}$ signaling function as more IHH molecules will interact with increased PTC1 and result in less SMO being released. As Npx did not affect the expression of GLI3, it is possible that GLI3 does not play a direct role in the osteogenesis of MSCs. Interestingly, we were unable to detect the expression of Sonic Hedgehog $(S H H)$, another gene involved in $\mathrm{HH}$ signaling and osteogenesis of MSCs, with primers that were specific for human $\mathrm{SHH}$.

The bone repair process in adults resembles normal development of the skeleton during embryogenesis. $\mathrm{Nu}-$ merous signaling pathways induce the osteogenic differentiation of MSCs. Although the mechanisms have not been fully discerned, and only $\mathrm{HH}$ signaling was studied in this research, other signaling pathways may also affect 
stem cell differentiation. As the results in this study indicate that Npx can affect treatment and lineage differentiation of MSC through the IHH signaling pathway, a better understanding of these underlying mechanisms and the effect of other NSAIDs on the osteogenesis of MSCs have far-reaching implications for improving bone repair for OA treatment.

\section{Conclusions}

The results support the concept that Npx has a dual role in that it can stimulate hypertrophic differentiation of MSCs, while suppressing osteogenic differentiation of MSCs and mineral deposition in the matrix through $\mathrm{HH}$ signaling.

\section{Abbreviations \\ ALP: alkaline phosphatase; bp: base pairs; COL1A1: collagen I, type 1; COL10A1: collagen X, type 1; Cpn: cyclopamine; DMEM: Dulbecco's modified Eagle's medium; FBS: fetal bovine serum; GAPDH: glyceraldehyde-3- phosphate dehydrogenase; GLI: GLI-Kruppel family member GLI; IHH: Indian hedgehog; MSC: mesenchymal stem cell; Npx: naproxen; NSAIDs: non- steroidal anti-inflammatory drugs; OA: osteoarthritis; OC: osteocalcin; OPN: osteopontin; PBS: phosphate-buffered saline; PCR: polymerase chain reaction; PTC1: Patched-1; SMO: Smoothened.}

\section{Competing interests}

The authors declare that they have no competing interests.

\section{Authors' contributions}

OS cultured the cells, performed the real-time RT-PCR, and analyzed the ALP activity. HTW designed the experiments, analyzed the data, and wrote the manuscript. AMA cultured the cells, and performed the real-time RT-PCR. IH and OC performed the real-time RT-PCR experiments and statistical analysis. RG cultured the cells and analyzed enzyme activity. JA designed the experiment, and revised the manuscript. FM conceived and supervised the whole study and finished writing the manuscript. All authors read and approved the final manuscript.

\section{Acknowledgements}

This study was supported by Canadian Institutes of Health Research (CIHR).

\section{Author details}

${ }^{1}$ Lady Davis Institute for Medical Research, Jewish General Hospital, McGill University, Montreal, Canada. ${ }^{2}$ Division of Orthopaedic Surgery, McGill University, Montreal, Canada. 'École Polytechnique de Montreal, Montreal, Canada. ${ }^{4}$ Orthopaedic Research Laboratory, Montreal General Hospital, McGill University, Montreal, Canada. ${ }^{5}$ Lunenfeld-Tanenbaum Research Institute at Mount Sinai Hospital, Toronto, Canada.

\section{Received: 1 March 2014 Accepted: 4 July 2014}

Published: 17 July 2014

\section{References}

1. Bijlsma JW, Berenbaum F, Lafeber FP: Osteoarthritis: an update with relevance for clinical practice. Lancet 2011, 377:2115-2126.

2. Richmond J, Hunter $D$, Irrgang J, Jones MH, Levy B, Marx R, Snyder-Mackler L, Watters WC, Haralson RH, Turkelson CM, Wies IL, Boyer KM, Anderson S, St Andre J, Sluka P, McGowan R: Treatment of osteoarthritis of the knee (nonarthroplasty). J Am Acad Orthop Surg 2009, 17:591-600.

3. Berger RG: Nonsteroidal anti-inflammatory drugs: making the right choices. J Am Acad Orthop Surg 1994, 2:255-260.

4. O'Connor JP, Lysz T: Celecoxib, NSAIDs and the skeleton. Drugs Today (Barc) 2008, 44:693-709.

5. Zeni JA Jr, Axe MJ, Snyder-Mackler L: Clinical predictors of elective total joint replacement in persons with end-stage knee osteoarthritis. BMC Musculoskelet Disord 2010, 11:86.
6. Li G, Yin J, Gao J, Cheng TS, Pavlos NJ, Zhang C, Zheng MH: Subchondral bone in osteoarthritis: insight into risk factors and microstructural changes. Arthritis Res Ther 2013, 15:R223.

7. Goldring MB, Goldring SR: Osteoarthritis. J Cell Physiol 2007, 213:626-634.

8. Vuolteenaho K, Moilanen T, Moilanen E: Non-steroidal anti-inflammatory drugs, cyclooxygenase-2 and the bone healing process. Basic Clin Pharmacol Toxicol 2008, 102:10-14.

9. Chang JK, Li CJ, Liao HJ, Wang CK, Wang GJ, Ho ML: Anti-inflammatory drugs suppress proliferation and induce apoptosis through altering expressions of cell cycle regulators and pro-apoptotic factors in cultured human osteoblasts. Toxicology 2009, 258:148-156.

10. Caplan Al, Bruder SP: Mesenchymal stem cells: building blocks for molecular medicine in the 21st century. Trends Mol Med 2001, 7:259-264.

11. Antoniou J, Wang HT, Alaseem AM, Haglund L, Roughley PJ, Mwale F: The effect of Link $N$ on differentiation of human bone marrow-derived mesenchymal stem cells. Arthritis Res Ther 2012, 14:R267.

12. Rosset $P$, Deschaseaux F, Layrolle P: Cell therapy for bone repair. Orthop Traumatol Surg Res 2014, 100:S107-S112.

13. Singh A, Goel SC, Gupta KK, Kumar M, Arun GR, Patil H, Kumaraswamy V, Jha S: The role of stem cells in osteoarthritis: an experimental study in rabbits. Bone Joint Res 2014, 3:32-37.

14. Almaawi A, Wang HT, Ciobanu O, Rowas SA, Rampersad S, Antoniou J, Mwale F: Effect of acetaminophen and nonsteroidal anti-inflammatory drugs on gene expression of mesenchymal stem cells. Tissue Eng Part A 2013, 19:1039-1046.

15. Rockel JS, Alman BA: Don't hedge your bets: hedgehog signaling as a central mediator of endochondral bone development and cartilage diseases. J Orthop Res 2011, 29:810-815.

16. Chung UI, Schipani E, McMahon AP, Kronenberg HM: Indian hedgehog couples chondrogenesis to osteogenesis in endochondral bone development. J Clin Invest 2001, 107:295-304.

17. Ruiz-Heiland G, Horn A, Zerr P, Hofstetter W, Baum W, Stock M, Distler JH, Nimmerjahn F, Schett G, Zwerina J: Blockade of the hedgehog pathway inhibits osteophyte formation in arthritis. Ann Rheum Dis 2012, 71:400-407.

18. Ayers KL, Thérond PP: Evaluating Smoothened as a G-protein-coupled receptor for Hedgehog signalling. Trends Cell Biol 2010, 20:287-298.

19. Claret S, Sanial M, Plessis A: Evidence for a novel feedback loop in the Hedgehog pathway involving Smoothened and Fused. Curr Biol 2007, 17:1326-1333.

20. Wijgerde M, McMahon JA, Rule M, McMahon AP: A direct requirement for Hedgehog signaling for normal specification of all ventral progenitor domains in the presumptive mammalian spinal cord. Genes Dev 2002, 16:2849-2864

21. Reichert JC, Schmalzl J, Prager P, Gillbert F, Quent VM, Steinert AF, Rudert M, Nöth U: Synergistic effect of Indian hedgehog and bone morphogenetic protein-2 gene transfer to increase the osteogenic potential of human mesenchymal stem cells. Stem Cell Res Ther 2013, 4:105.

22. Briscoe J, Thérond PP: The mechanisms of Hedgehog signalling and its roles in development and disease. Nat Rev Mol Cell Biol 2013, 14:416-429.

23. James AW: Review of signaling pathways governing MSC osteogenic and adipogenic differentiation. Scientifica (Cairo) 2013, 2013:684736.

24. Mwale F, Girard-Lauriault PL, Wang HT, Lerouge S, Antoniou J, Wertheimer MR Suppression of genes related to hypertrophy and osteogenesis in committed human mesenchymal stem cells cultured on novel nitrogen-rich plasma polymer coatings. Tissue Eng 2006, 12:2639-2647.

25. Tchetina E, Mwale F, Poole AR: Distinct phases of coordinated early and late gene expression in growth plate chondrocytes in relationship to cell proliferation, matrix assembly, remodeling, and cell differentiation. J Bone Miner Res 2003, 18:844-851.

26. Shum L, Nuckolls $\mathrm{G}$ : The life cycle of chondrocytes in the developing skeleton. Arthritis Res 2002, 4:94-106.

27. Gould A, Missailidis S: Targeting the hedgehog pathway: the development of cyclopamine and the development of anti-cancer drugs targeting the hedgehog pathway. Mini Rev Med Chem 2011, 11:200-213.

28. Bar EE, Chaudhry A, Lin A, Fan X, Schreck K, Matsui W, Piccirillo S, Vescovi AL, DiMeco F, Olivi A, Eberhart CG: Cyclopamine-mediated hedgehog pathway inhibition depletes stem-like cancer cells in glioblastoma. Stem Cells 2007, 25:2524-2533 
29. Yoon DS, Yoo JH, Kim YH, Paik S, Han CD, Lee JW: The effects of COX-2 inhibitor during osteogenic differentiation of bone marrow-derived human mesenchymal stem cells. Stem Cells Dev 2010, 19:1523-1533.

30. Pountos I, Giannoudis PV, Jones E, English A, Churchman S, Field S, Ponchel F, Bird H, Emery P, McGonagle D: NSAIDS inhibit in vitro MSC chondrogenesis but not osteogenesis: implications for mechanism of bone formation inhibition in man. J Cell Mol Med 2011, 15:525-534.

31. Styner M, Sen B, Xie ZH, Case N, Rubin J: Indomethacin promotes adipogenesis of mesenchymal stem cells through a cyclooxygenase independent mechanism. J Cell Biochem 2010, 111:1042-1050.

32. Motawi TM, Bustanji Y, El-Maraghy SA, Taha MO, Ghussein MA: Naproxen and cromolyn as new glycogen synthase kinase $3 \beta$ inhibitors for amelioration of diabetes and obesity: an investigation by docking simulation and subsequent in vitro/in vivo biochemical evaluation. J Biochem Mol Toxicol 2013, 27:425-436.

33. Kozhemyakina E, Cohen T, Yao TP, Lassar AB: Parathyroid hormone-related peptide represses chondrocyte hypertrophy through a protein phosphatase 2A/histone deacetylase 4/MEF2 pathway. Mol Cell Biol 2009, 29:5751-5762.

34. Li Y, Ahrens MJ, Wu A, Liu J, Dudley AT: Calcium/calmodulin-dependent protein kinase II activity regulates the proliferative potential of growth plate chondrocytes. Development 2011, 138:359-370.

doi:10.1186/ar4614

Cite this article as: Salem et al:: Naproxen affects osteogenesis of human mesenchymal stem cells via regulation of Indian hedgehog signaling molecules. Arthritis Research \& Therapy 2014 16:R152.

\section{Submit your next manuscript to BioMed Central and take full advantage of:}

- Convenient online submission

- Thorough peer review

- No space constraints or color figure charges

- Immediate publication on acceptance

- Inclusion in PubMed, CAS, Scopus and Google Scholar

- Research which is freely available for redistribution 